\title{
Determination of an Effective Wedge Angle by Combination of Two Arbitrary Universal Wedge Fields in Radiation Therapy of Cancer Patients with Megavoltage Photon Beams
}

\author{
Azin Shamsi ${ }^{1,2}$, Mohammad Javad Tahmasebi Birgani ${ }^{1,2 *}$, Mohammad Ali \\ Behrooz $^{1}$, Sholeh Arvandi ${ }^{1,2}$, Jafar Fatahiasl ${ }^{1}$, Reza Maskny ${ }^{2}$, Neda Abdalvand ${ }^{1,2}$
}

\begin{abstract}
Background: Wedge filters are commonly used in radiation oncology for eliminating hot spots and creating a uniform dose distribution in optimizing isodose curves in the target volume for clinical aspects. These are some limited standard physical wedges $\left(15^{\circ}, 3^{\circ}, 4^{\circ}, 6^{\circ}\right)$,or creating an arbitrary wedge angle, like motorized wedge or dynamic wedge,... The new formulation is presented by the combination of wedge fields for determining an arbitrary effective wedge angles. The isodose curves also are derived for these wedges. Materials and Methods: we performed the dosimetry of Varian Clinac 2100C/D with Scanditronix Wellhofer water blue phantom, CU500E, OmniPro - Accept software and $0.13 \mathrm{cc}$ ionization chamber for $6 \mathrm{Mv}$ photon beam in depth of $10 \mathrm{~cm}$ (reference depth) for universal physical wedges $\left(15^{\circ}, 30^{\circ}, 45^{\circ}\right.$, and $\left.60^{\circ}\right)$ and reference field $10.10 \mathrm{~cm}^{2}$. By combining the isodose curve standard wedge fields with compatible weighting dose for each field, the effective isodose curve is calculated for any wedge angle. Results: The relation between a given effective wedge angle and the weighting of each combining wedge fields was derived. A good agreement was found between the measured and calculated wedge angles and the maximum deviation did not exceed $3^{\circ}$. The difference between the measured and calculated data decreased when the combined wedge angles were closer. The results are in agreement with the motorized single wedge appliance in the literature. Conclusions: This technique showed that the effective wedge angle that is obtained from this method is adequate for clinical applications and the motorized wedge formalism is a special case of this consideration.
\end{abstract}

Keywords: Universal physical wedge - profile - megavoltage photon beam - radiation therapy

Asian Pac J Cancer Prev, 17 (1), 197-200

\section{Introduction}

One of the methods of treating of cancerous patients is radiation therapy in the form of curative or palliative by using a Co-teletherapy machine or linear accelerators(Abrath and Purdy, 1980). Acceptable dose uniformity is within $\pm 5 \%$ in radiation therapy. wedge filters are commonly used for uniforming, modifying or optimizing isodose distribution in the target volume for better dose delivery (Khan and Gibbons, 2014). Wedge filters are of the several types like physical or dynamical. The physical wedge is usually made of dense materials such as lead or steel and is mounted on a tray and inserted in the radiation field at a specified distance from the patient. Dynamical wedge generated electronically through the motion of independent jaws within the treatment region. Most accelerators are provided with a selection of wedges that are mounted externally on the head of the machine. The wedge isodose angle has been defined as the angle between the isodose curve passing through reference depth and the normal horizontal line on the central axis on this depth. According to the international commission on radiation units and Measurements (ICRU), the path of the radiation beam decreases the dose rate and this must be taken into account in dose calculation by a wedge transmission factor, defined as the ratio of dose in water at a point on a central axis with and without the wedge. In some systems of radiation therapy a single universal wedge maybe used that mounted inside the head and moved by remote control and is named by motorized wedge (Bentel et al., 1982). For single 600 motorized wedge, Paula and et al. (1985) explained a new method for obtaining an effective wedge angle by combining an open field with a 600 motorized wedge with specified weighting doses that can be determined as the ratio of the slopes of the central-axis depth dose curves for open and wedge fields(Petti and Siddon, 1985). For a motorized wedge, Rajesh Kumar and etal. (2011) used an analytical approach

${ }^{1}$ Department of Medical physics Faculty of medical sciences Ahwaz jondishapour university of medical sciences, ${ }^{2}$ Departments of Clinical Oncology, Golestan Hospital, Ahvaz Jundishapur University of Medical Sciences, Ahvaz, Iran Ahvaz, Iran *For correspondence:Tahmasebi_mj @yahoo.com 
for an arbitrary effective wedge angle.The relationship was established between the wedge beam weight and effective wedge angle (Kumar et al., 2012). Inhikar Rajesh and et al. (2007) estimated the transit dose for motorized wedge treatment in the Equinox telecobalt machine. The actual measurements carried out with ion chamber for universal wedge and motorized wedge showed no variation between the dose delivered with TLD measurement (Kinhikar et al., 2007b). The motorized wedge filter has a number of advantages, like no probability of physical injury to operators and patient; no need to handle physical wedges; making wedge selection faster and easier, which results in higher patients throughout and less fatigue for operators and flexibility to generate arbitrary wedge angle instead of the limited standard angles. Clinical commissioning of a motorized wedge filter for a Theratron Equinox telecobalt unit has been reported in the literatures (Kinhikar et al., 2007a; Sahani et al., 2009).

Tamer Dawod (2015) evaluated the motorized wedge supported by the dosimetric performance 3-D treatment planning system for Electa Precise linear accelerator by comparing the calculated and measured doses for symmetric and asymmetric wedge fields in depth of d_max, $5 \mathrm{~cm}, 10 \mathrm{~cm}, 20 \mathrm{~cm}$ in water phantom for $6 \mathrm{mv}$ and $15 \mathrm{mv}$ photon beams. By using $0.125 \mathrm{cc}$ ionization chamber a good agreement was found between calculated and measured dose. Maximum deviation was not longer than \%5. This deviation for a symmetric field was less than asymmetric and increased with increasing wedge angle. The result of this study showed that Electa precise with motorized wedge for symmetric and asymmetric field is adequate for the clinical applications. In 2007, Rajesh A. Kinhikar et al. evaluated thetelecobaltTheratron Equinox-80 with a single motorized wedge. They configured Eclipes 3-D treatment planning(Varian PaloAlto,USA)for universal wedges $\left(15^{\circ}, 30^{\circ}, 45^{\circ}\right.$, and $60^{\circ}$ ) and their profile and central axis depth doses were measured with blue water phantom and compared with actual universal wedges in a homogeneous phantom generated in Eclipse for various field sizes. The variation in measured and calculated dose at $10 \mathrm{~cm}$ depth was within $\% 2$ and angle within $2^{\circ}$. Then, the motorized wedge was successfully configured in Eclipse for four the above wedge angles (Kinhikar et al., 2007b). In 2014, SJ-Weston et al. evaluated six Electa Precise Linac creating an arbitrary angle by a combination of open and motorized wedge with specified weightings for definite dose delivery. They found a range of $\% 4$ in measured transition factor for $6 \mathrm{mv}$ photon beams (Weston et al., 2014). The aim of this study was the consideration of combining two specified wedge shape fields with determined weighting and containing an arbitrary effective wedge shape distribution and effective wedge in the clinical radiation therapy.

\section{Materials and Methods}

\section{Theory}

For determination of an arbitrary effective wedge angle $\theta$ by combining the standard, universal wedge angles $\phi$ and $\phi$, one can obtain a weighting factor for this combination from the isodose lines or profiles of these wedge shaped fields in the reference depth $10 \mathrm{~cm}$.For this purpose, profiles and isodose lines combining two wedged shape fields are drawn in the blue water phantom for depth $10 \mathrm{~cm}$ by $0.13 \mathrm{cc}$ ionization chamber as shown fig. 1 .

According to Figure 1, one can write:

$\tan \phi=M N / U=(O N-O M) / U$,
$\tan \phi=A B / U=(O B-O A) / U$,

If the wedge waithings of two fields are respectively $\alpha$ and 1- $\alpha$, multiplaying both sides of Eq. (1-b)by $\alpha$ and Eq.(1-c)by $(1-\alpha)$, gives

$\alpha \tan \phi=(\alpha((\alpha(O A)-(O A) / U$,

$(1-\alpha) \tan \phi^{\prime}=\left((1-\alpha) O B^{\prime}-(1-\alpha) O A^{\prime}\right) / U$

by combining two Eqs.(2a)and(2b), according to Figure

1 , one can obtain

$\alpha \tan \phi+(1-\alpha) \tan \phi^{\prime}=\left[\left(\alpha O B+(1-\alpha) O B^{\prime}\right) / U\right]-[(\alpha O A+(1-\alpha)$

$\left.\left.\boldsymbol{O A}^{\prime}\right) / \boldsymbol{U}\right]$.

Or

$\alpha \tan \phi+(1-\alpha) \tan \phi^{\prime}=[(O N-O M) / U]=\tan \theta$

By rewriting the Eq.(4), the weighting factor can becomes $\alpha=\left(\tan \theta-\tan \phi^{\prime}\right) /\left(\tan \phi-\tan \phi^{\prime}\right)$

From fig.1 one can

$\alpha(O A)+(1-\alpha) O A^{\prime}=O M$,

Where $\mathrm{OA}, \mathrm{OA}^{\prime}$ and $\mathrm{OM}$ are profiles of combining wedge-shapedfields. This means that

$P D D \_\theta=\alpha P D D \_\phi+(1-\alpha) P D D \_\left(\phi^{\prime}\right)$.

\section{Measurements}

Relative dosimetry is done for obtaining PDD, profile and isodose curves. Using scanditronix Welhopher water blue phantom, MCU500EU, OminoPro_Accept software and $0.13 \mathrm{cc}$ ionization chamber for $6 \mathrm{MV}$ photon beam of varian $2100 \mathrm{C} / \mathrm{D}$ linac in depthes of $1,4,7,10,13 \mathrm{~cm}$ of $10 \mathrm{~cm}$ for universal physical wedges $\left(15^{\circ}, 30^{\circ}, 45^{\circ}\right.$, and $\left.60^{\circ}\right)$ and reference field of $10.10 \mathrm{~cm}^{2}$, PDD, profiles and isodose lines curves are plotted.

\section{Results}

Using ScanditronixWelhopher water blue phantom, CU500E, OmniPro - Accept software and 0.13cc ionization chamber for $6 \mathrm{MV}$ photon beam of varian2100C/D linac the percentage of depth dose curves, profiles (for 10.10 $\mathrm{cm}^{2}$ wedge fields $0^{\circ}, 15^{\circ}, 45^{\circ}$ and $60^{\circ}$ in depths of $1,4,7$, 10 , and $13 \mathrm{~cm}$ are obtained from the relative dosimetry. Combining the profiles gives isodose curves. For example,

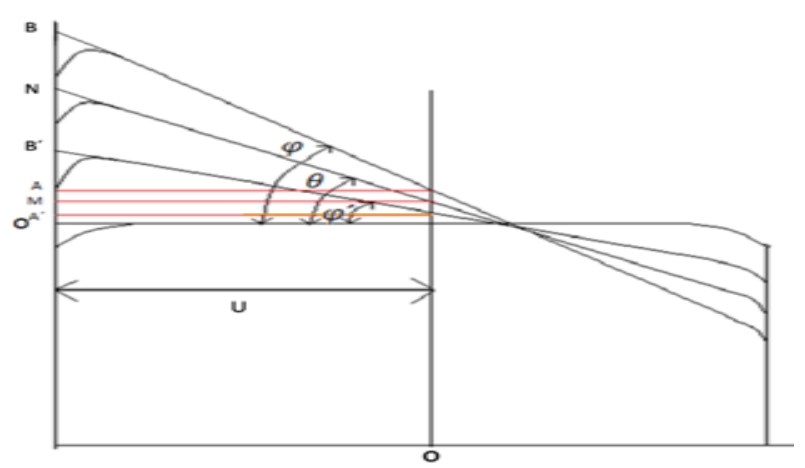

Figure 1. Combination of the Isodose Curves of two Wedges Fields with Angles $\phi$ and $\phi^{\prime}$ and Generating the Desired Wedge Field with Angle 


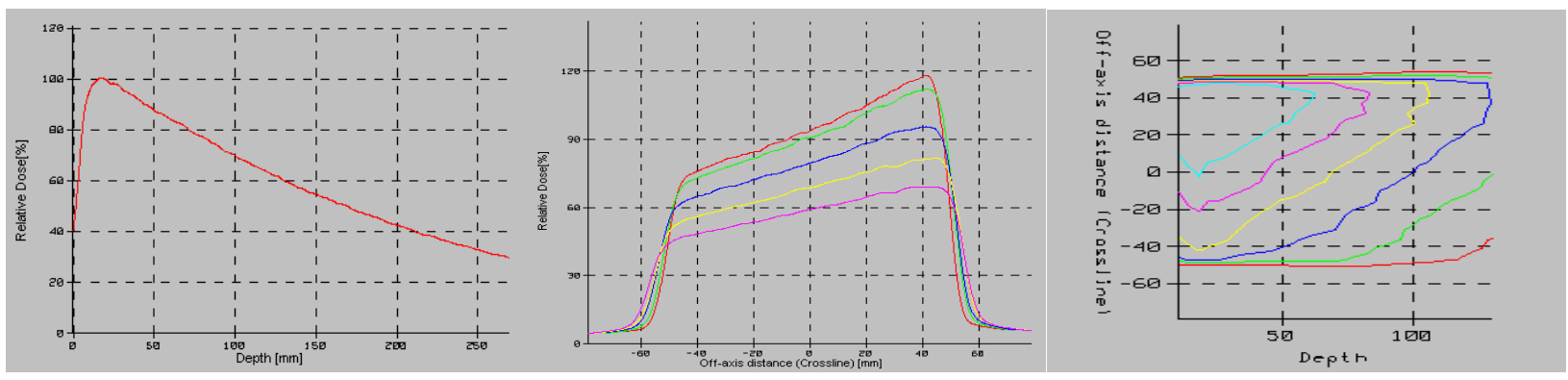

Figure 2. A). PDD for $45^{\circ}$,wedge shape field $10 \mathrm{~cm} .10 \mathrm{~cm}$; B). Profile for $45^{\circ}$ wedge shaped field $10 \mathrm{~cm} .10 \mathrm{~cm}$ in

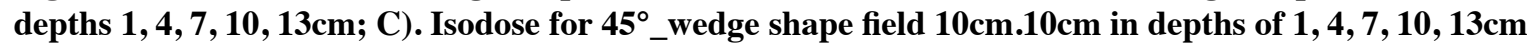

Table 1. The Combination of two Wedges Fields $\left(15^{\circ}, 30^{\circ}, 45^{\circ}\right.$, and $\left.60^{\circ}\right)$ with Weighing $\% 40$ and $\% 60$ and Generated Effective wedge Angle in Comparison with Measurement

\begin{tabular}{|c|c|c|c|c|c|c|c|c|c|c|}
\hline \multirow[t]{2}{*}{$40 / 60$} & \multicolumn{2}{|c|}{60} & \multicolumn{2}{|c|}{45} & \multicolumn{2}{|c|}{30} & \multicolumn{2}{|c|}{15} & \multicolumn{2}{|c|}{0} \\
\hline & measurement & Analytical & measurement & Analytical & measurement & Analytical & measurement & Analytical & measurement & Analytical \\
\hline 0 & 40.9 & 43.7 & 28.1 & 30.96 & 21.4 & 21.3 & 10.7 & 11 & & \\
\hline 15 & 47.2 & 47.3 & 35.4 & 36.05 & 27.6 & 27.4 & & & 9.4 & 7.4 \\
\hline 30 & 51.3 & 50.6 & 41.3 & 40.7 & & & 26.6 & 24.3 & 14 & 14.7 \\
\hline 45 & 52.7 & 53.6 & & & 36.6 & 38.3 & 29.7 & 30.6 & 21.8 & 21.8 \\
\hline 60 & & & 50.4 & 51.1 & 46.9 & 45.8 & 39.2 & 39.7 & 32.1 & 32.5 \\
\hline
\end{tabular}

Table 2. The Combination of two Wedges Fields $\left(15^{\circ}, 30^{\circ}, 45^{\circ}\right.$, and $\left.60^{\circ}\right)$ with Weighing $\% 20$ and $\% 80$ and Generated Effective wedge Angle in Comparison with Measurement

\begin{tabular}{|c|c|c|c|c|c|c|c|c|c|c|}
\hline \multirow[t]{2}{*}{$20 / 80$} & \multicolumn{2}{|c|}{60} & \multicolumn{2}{|c|}{45} & \multicolumn{2}{|c|}{30} & \multicolumn{2}{|c|}{15} & \multicolumn{2}{|c|}{0} \\
\hline & measurement & Analytical & measurement & Analytical & measurement & Analytical & measurement & $\overline{\text { Analytical }}$ & measurement & Analytical \\
\hline 0 & 51.2 & 51.9 & 37.5 & 38.6 & 28.3 & 27.45 & 12.3 & 14.6 & & \\
\hline 15 & 53.3 & 53.2 & 41.5 & 40.8 & 31.5 & 30.3 & & & 5.2 & 3.7 \\
\hline 30 & 55.1 & 54.6 & 43.8 & 42.9 & & & 20 & 21.1 & 9.4 & 7.4 \\
\hline 45 & 52.7 & 53.6 & & & 35.3 & 35.75 & 21.9 & 24.5 & 13.2 & 11.3 \\
\hline 60 & & & 48.8 & 48.2 & 39.5 & 39.99 & 30.2 & 29.85 & 18 & 17.7 \\
\hline
\end{tabular}

these curves a given or $10.10 \mathrm{~cm}^{2}, 45^{\circ}$ wedge angle fields in Figures. 2 (a, b, c).

For two arbitrary weighting $\alpha=20 \%$ and $\alpha=40 \%$, the Eq.7 is examined. By combining four standard wedges $\left(15^{\circ}, 30^{\circ}, 45^{\circ}\right.$, and $\left.60^{\circ}\right)$ and open fields from Eqs. (5 and 7 ) the effective angle $\theta$ are derived for two above values $\alpha$ and tabulated in tables 1 and 2

By using Eq.7 for these two values the related profiles are combined and plotted by the OmniPro software and the slope of these effective wedge angle profiles are measured and compared with analytical angle tabulated in tables 1 and 2 .

\section{Discussion}

In this study an analytical method for generating an effective wedge arising of a combination of two physical wedges (PW) with any weighting is presented and compared with measurements and other researches.

Paula and Siddon describe a technique for determining an effective wedge angle from a combination of open and motorized $60^{\circ}$ wedge angle fields with data from the Philips.SL/75-6MV accelerator by weighting factor parameter that interpreted physically as the ratio of the slopes of the central depth dose curves of the effective motorized wedge fields. Their results were consistent with the data within $3^{\circ}$ (Petti and Siddon, 1985).

Jingeng Zhu introduces a new method for generating wedge- shaped dose distribution through dynamic multileaf collimator dose delivery rather than computer controlled jaw motion. His method tested by the shape of standard wedges $\left(15^{\circ}, 30^{\circ}, 45^{\circ}\right.$, and $\left.60^{\circ}\right)$ the maximum deviation is within $2.8 \%$ (Zhu, 2005).

By a combination of physical motorized wedge field and open field with different weighing factors, Rajesh et al. generated the desired wedge angle -shaped $\left(0^{\circ}\right.$ to $60^{\circ}$ ) field. Their formula is similar to Eq.5 for $\phi^{\prime}=0$ and therefore is a special case of our formalism of the measured and calculated MV telecobalt Equinox_80. The obtained angles are well agreed within $2^{\circ}$ (Kinhikar et al., 2007b).With use of an enhanced dynamic wedge (EDW) technique, BajusovaAlica in ( 2009) for determined an arbitrary wedge angle for linear accelerator CLINAC $600 \mathrm{C} / \mathrm{D}$ generating $6 \mathrm{Mv}$ beams of $\mathrm{X}$-ray. in this technique, the deviation between the calculated and measured dose value is lower than 1.5 (Bajusová et al., 2010).

In a similar research in 2010, Misbah Ahmad et al. reported maximum variation of $8.9 \%$ between EDW and PWs. Rajesh Kumar and et al for pland motorized wedge angles in different fields reported difference between planed and measured wedge angles less than $2^{\circ}$.

In another similar study, Sathiyan Saminathan et al., in 2012 in Greater Poland Cancer Centre the dosimetric properties of varian EDW and PW were analyzed and compared in $6 \mathrm{MV}$ photons. The maximum variation in the output factor is between $1.5 \%-3.8 \%$ for all fields and EDW has a higher wedge factor compared to that of the physical wedge for a particular wedge angle(Saminathan 
et al., 2012). In this research the wedged angle obtained by the presented formalism and measurement was less than $3^{\circ}$ that is comparable with the studies of Tamer Dawod on the motorized 60 wedge angle of 6MV Electa Precise linac (Saminathan et al., 2012). furthermore, it is consistent with the method analytically performed by Petti and Siddon for effective wedge angles with a $60^{\circ}$ _universal motorized wedge that by comparison with measurement had a deviation within $3^{\circ}$ over the range of wedge angle and field size(Petti and Siddon, 1985). In our research the maximum deviation with measurement are less than $3^{\circ}$ as shown in table 1 and 2.

In conclusion, the effective wedge angle obtained by a combination of the universal wedge fields for clinical applications is exactly acceptable and in agreement with measured data.

\section{Acknowledgements}

This work is Shamsi,s thesis and was supported by Ahvaz jundishapur University of Medical Sciences, Ahvaz, Iran(Grant Number B_9427).

\section{References}

Abrath FG, Purdy JA (1980). Wedge design and dosimetry for 25-MV x rays. Radiology, 136, 757-62.

Bajusová A, Králik G, Miglierini M (2010). Means of Intensity Modulation of Radiation in External Radiotherapy. Nuclear Physics Methods And Accelerators In Biology And Medicine: Fifth International Summer School on Nuclear Physics Methods and Accelerators in Biology and Medicine. AIP Publishing, 195-7.

Bentel G, Nelson C, Noell K (1982). Treatment planning \&. dose calculation in radiation.

Khan FM, Gibbons JP 2014. Khan's the Physics of Radiation Therapy, Lippincott Williams \& Wilkins.

Kinhikar R, Sharma S, Upreti R, et al (2007a). Commissioning of motorized wedge for the first Equinox-80 telecobalt unit and implementation in the Eclipse 3D treatment planning system. Australasian Physics \& Engineering Sciences in Medicine, 30, 127-34.

Kinhikar RA, Sharma S, Upreti R, et al (2007b). Characterizing and configuring motorized wedge for a new generation telecobalt machine in a treatment planning system. Journal of medical physics/Association of Medical Physicists of India, 32, 29.

Kumar R, Kar D, Sharma S, et al (2012). Design, implementation and validation of a motorized wedge filter for a telecobalt machine (Bhabhatron-II). Physica Medica, 28, 54-60.

Petti PL, Siddon RL (1985). Effective wedge angles with a universal wedge. Physics in medicine and biology, 30, 985.

Sahani G, Kumar M, Sharma PD, et al (2009). Compliance of Bhabhatron-II telecobalt unit with IEC standard-Radiation safety. Journal of Applied Clinical Medical Physics, 10.

Saminathan S, Manickam R, Supe SS (2012). Comparison of dosimetric characteristics of physical and enhanced dynamic wedges. Reports of Practical Oncology \& Radiotherapy, 17, 4-12.

Weston S, Thompson R, Morgan A (2014). The matching of wedge transmission factors across six multi-energy linear accelerators. The British journal of radiology.

Zhu J (2005). Generation of wedge-shaped dose distributions through dynamic multileaf collimator dose delivery. Journal of Applied Clinical Medical Physics, 6.

200 Asian Pacific Journal of Cancer Prevention, Vol 17, 2016 